\title{
Multiple Sink Based Compressive DATA Aggregation TECHNIQUE FOR WIRELESS SENSOR NETWORK
}

\author{
Mohamed Yacoab M.Y. \\ Research Scholar, Karpagam University, Coimbatore, India \\ MEASI Institute of Information Technology, Chennai, India \\ yacoab@yahoo.com \\ Dr.V.Sundaram \\ Director, Karpagam College of Engg, Coimbatore, India \\ dr_vsundarameyahoo.com
}

\begin{abstract}
In a wireless sensor network, the single sink based data aggregation technique leads to inefficiency due to imbalance in energy consumption. Moreover it induces scalability problems and overload at the sink, since it is a "many to one" pattern. Hence, in this paper, we develop a multiple sink based data aggregation technique, assuming the sinks are static. In this technique, initially a sink oriented tree is determined for each sink. If the amount of data in the network becomes large, the data is transmitted in the slots allocated for the specific part of the data such that interference is avoided in the data transmission. As data gets aggregated at the nodes which are nearer to the sink it will be compressed and then forwarded to the next level. This way data is efficiently transmitted to the sink without any loss and interference. By simulation results, we show that our proposed technique achieves good packet delivery ratio with reduced energy consumption and delay.
\end{abstract}

KEYWORDS : Data Aggregation, Compressive, Multiple Sinks

\section{INTRODUCTION}

\subsection{Wireless Sensor Network}

A wireless sensor network is an upcoming technology which is being given major consideration by the research community. Several small, low cost devices constitute the sensor network which is actually a self-organizing ad-hoc system. Its main function is monitoring the physical environment and consequently collects and dispatch information to one or more sink nodes [1]. In wireless sensor network, the main operations performed are related to monitoring the physical environment, sensed information processing and result delivery to the particular sink nodes. To perform these tasks, the sensor nodes are powered by the batteries which are resources of limited energy. Hence, designing an energy efficient protocol for increasing the network lifetime is the major dispute in this energy constrained system [2]. 
International Journal of Wireless \& Mobile Networks (IJWMN) Vol. 3, No. 2, April 2011

\subsection{Data Aggregation}

A common function of the sensor network is data gathering. Here the sampled information at each of the sensor nodes has to be transferred, for the sake of further processing and analysis to the central base station [3]. In wireless sensor networks, data aggregation is considered as the most primary distributed data processing procedures which saves energy and reduces medium access layer contention [4]. For wireless routing in sensor networks, data aggregation is considered as a vital paradigm. It involves merging the data from various sources along the route avoiding the redundancy, reducing the transmission numbers and hence saving the energy. Thus the focus is shifted from the customary address-centric approach for networking to a more data-centric approach [5].

\subsection{Issues related to the Single Sink Based Data Aggregation}

1] In sensor data gathering, the "many-to-one" traffic pattern may lead to imbalance in energy expenditure to a higher extent in the complete network, causing early termination of the network lifetime. Hence the open challenge is to extend the network lifetime by balancing the energy consumption and at the same time maintaining energy efficiency [8].

2] Scalability problem is seen in the single sink network architecture. In single sink architecture, the data aggregated at the sink may become more than its communication capacity due to the large number of sensors in the network. In addition, the radio channel capacity close to the sink may become overloaded if the average number of hops between the source and the sink becomes excess [10].

3] In single sink network architectures, in case the sink is heavily loaded, then the data will not reach the destination, resulting in transmission failure.

\subsection{Multi Sink based Data Aggregation}

The scalability of sensor networks increases with the introduction of the multiple sinks [11]. By a pseudo link, all sink nodes are connected to pseudo destination. In multiple sink network which in turn changes it to a single reverse multicast tree [12]. Efficient data gathering trees are formed by the nodes and then the best sink is chosen for transferring the data in multiple sink networks. The mean distance between the nodes and the sink will be decreased in the multi sink network leading to energy savings and increased lifetime. The sink acts as a gateway in multi sink network, forwarding the sensed data towards the storage system in the network as in the case of internet. Only the data produced by a particular set of devices is collected by the sink and then the entire phenomenon which is monitored will be reconstructed at the data storage system [10].

\subsection{Previous Works}

In our first work [6], we have proposed an adaptive traffic aware aggregation technique for wireless sensor networks. A traffic monitoring agent is used to monitor the load status of the event traffic. If the total traffic load of the system is less than a threshold value, then the structured lossless aggregation is applied. When the traffic load crosses the threshold, then the aggregation technique is adaptively changed to structure-free lossy aggregation. 
In our second work [7] as an extension of the first work, we provide a cost effective compressive data gathering technique to enhance the traffic load, by using structured data aggregation scheme. The use of compressive data gathering process provides a compressed sensor reading to reduce global data traffic and distributes energy consumption evenly to prolong the network lifetime.

In this work as an extension to our previous two works, we propose to develop an effective data aggregation technique which includes multiple sinks in WSN.

\section{RELATED WORK}

Khalid N Chaaran et al [13] proposed a scheme in which the all nodes except sinks, act also as message forwarding nodes. These messages are received from any one of the neighbor nodes and are needed to be forwarded to one or many neighbor nodes. The forwarding decision is based on node's own knowledge, sender's guidance and neighborhood knowledge. The ultimate goal, while making these forwarding decisions at nodes, is to find shortest possible route with maximum path aggregation but not at the cost of delay. A scalable multi-path routing approach called Neighbor Sink Nexus (NSN) routing algorithm is presented in this paper to address propagation energy constraints. Security features are not incorporated in this technique and robustness need to be enhanced.

Luca Mottola et al [14] in this paper presented MUSTER (MUlti-Source MUlti-Sink Trees for Energy-efficient Routing), a routing protocol expressly designed for many-to-many communication. First, we devise an analytical model to compute, in a centralized manner, the optimal solution to the problem of simultaneously routing from multiple sources to multiple sinks. Next, we illustrate heuristics approximating the optimal solution in a distributed setting, and their implementation in MUSTER. To increase network lifetime, MUSTER minimizes the number of nodes involved in many-to -many routing and balances their forwarding load. This technique results in uneven energy consumption when the nodes along merged paths experience an increased routing load.

Sixia Chen et al [15] in this paper considered Multiple-Sink Data Collection Problem in wireless sensor networks, where a large amount of data from sensor nodes needs to be transmitted to one of multiple sinks. An approximation algorithm is designed to minimize the latency of data collection schedule and show that it gives a constant-factor performance guarantee. A heuristic algorithm is also presented based on breadth first search for this problem.

Waleed Alsalih et al [16] in this paper proposed a mobile base station placement scheme for extending the lifetime of the network. In our scheme the life of the network is divided into rounds and base stations are moved to new locations at the beginning of each round. In this paper, a more general problem in which a base station can be placed anywhere in the sensing field is defined and solved. The problem is formulated as an Integer Linear Program (ILP) and an ILP solver (with a constant time limit) is used to find a near-optimal placement of the base stations and to find routing patterns to deliver collected data to base stations.

Zoltan Vincze et al [17] in this paper give a mathematical model that determines the locations of the sinks minimizing the sensors average distance from the nearest sink. First an iterative algorithm called global that is able to find the sink locations given by the mathematical model is presented. However, it uses global information about the network that is impractical in 
wide area sensor networks, thus they have proposed a novel iterative algorithm called lhop that carries out the sink deployment based only on the location information of the neighboring nodes while the location of the distant nodes is being approximated. The two algorithms are compared and show that 1hop approaches the performance of global very closely. Another important issue is that the neighboring nodes of the sinks have a high traffic load, thus the lifetime of the network can be elongated by relocating the sinks from time to time. Based on the 1hop algorithm they have proposed the 1hop relocation algorithm for the coordinated relocation of multiple sinks.

\section{Proposed Work}

\subsection{Compressive Data Gathering}

In our previous paper [7], we have proposed a compressed data gathering technique which is adopted in this paper for the transmission of the data towards multiple sinks. The perception behind CDG in our previous work is that joint transmission of the correlated sensor readings instead of transmission of the readings separately will increase the efficiency to a higher level. On the sub tree basis data gathering and reconstruction of the CDG are performed.

We assume that the multiple sinks in the network are static. Each node should know its local routing structure so as to combine the sensor readings when it is being transmitted. That is, if the given node in the routing tree is a leaf node or not or if the node is an inner node then how many children does it have. To the standard routing protocol, a small modification is done so as to facilitate proficient aggregation: when a parent node is chosen by the node, it transmits a "subscribe notification" to that node and an "unsubscribe notification" is sent to the old parent, when the node changes the parent.

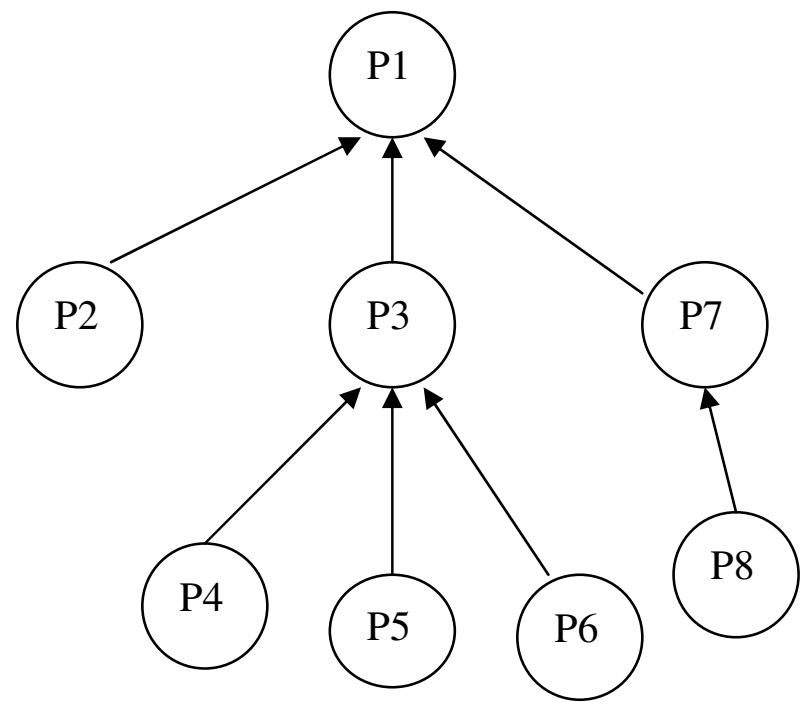

Fig.1 data gathering process of CDG

The example shown in fig. 1 illustrates the data gathering process of CDG. The leaf nodes will initiate the transmission only after all nodes receive their readings.

In this example, a random number $\beta_{i 2}$ is generated by $\mathrm{P} 2$ and it computes $\beta_{i 2} u_{2}$ and then the value is sent to $\mathrm{P} 1$. The ith weighted sum is denoted by the index $\mathrm{i}$ which ranges from 1 to 
M. Likewise $\beta_{i 4} u_{4}, \beta_{i 5} u_{5}$ and $\beta_{i 6} u_{6}$ is transmitted to P3 by P4, P5 and P6. After the three values are received by $\mathrm{P} 3$ it will compute the value $\beta_{i 3} u_{3}$ and then it adds to the sum of the relayed value. It then transmits to $\mathrm{P} 1$ the value. Next $\boldsymbol{\beta}_{i 1} \boldsymbol{u}_{1}$ is computed by the node $\mathrm{P} 1$ and is transmitted. Lastly, to the sink, the message which contains the weighted sum of all readings in a sub tree is forwarded.

In a specific tree, if it is assumed to have $\mathrm{N}$ nodes and $\mathrm{M}$ measurements are intended to be collected by the sink. Then regardless of the hop distance of the node to the sink, all nodes will send the same number of $O(M) . O(N M)$ will be the overall message complexity. If $\mathrm{M} \ll \mathrm{N}$, then less messages are transmitted by CDG when compared with the baseline data collection when $O\left(N^{2}\right)$ is the worst case message complexity. More importantly, for the extension of the lifetime of the bottleneck sensors as well as the entire network, the transmission load is spread uniformly.

The $\mathrm{i}^{\text {th }}$ weighted sum can be represented by:

$$
U_{i}=\sum_{j=1}^{N} \beta_{i j} u_{j}
$$

The sink obtains $\mathrm{M}$ weighted sums $\left\{\mathrm{U}_{\mathrm{i}}\right\}, \mathrm{i}=1,2 \ldots \mathrm{M}$. Mathematically, we have:

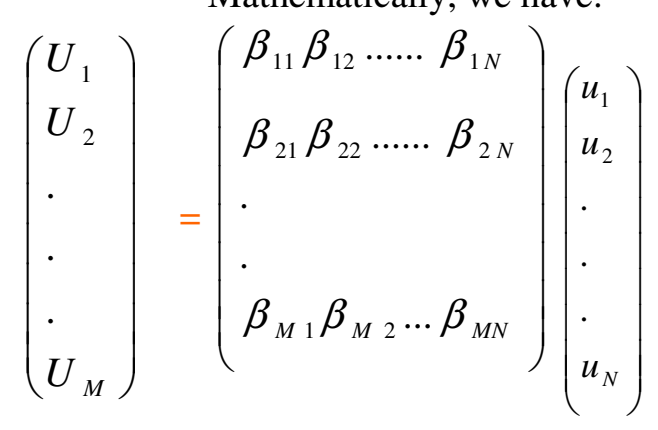

In the above equation, series of random numbers are placed in each column of $\left\{\beta_{i j}\right\}$ which is produced at the corresponding node. A simple strategy is used for preventing the transmission of the random matrix from sensors to the sink: a random seed is broadcasted to the entire network before transmission. Using this global seed and its unique identification, each sensor will generate its own seed. Each sensor generates a corresponding series of coefficients from a pre-installed pseudo random number generator. Given that the sink knows the identifications of all sensors, the coefficients can be reproduced at the sink.

In (2), $\boldsymbol{u}_{\boldsymbol{i}}(\mathrm{i}=1,2 \ldots \mathrm{N})$ is a scalar value. Each node is possibly attached with a few sensors of different type, e.g., temperature sensor and a humidity sensor in a practical sensor network. Then from each node, the sensor readings become a multi dimensional vector. In this case, in each dimension we may separate the readings and process them. Alternatively, since for the sensor readings, the random coefficients $\beta_{i j}$ are irrelevant, $\boldsymbol{u}_{i}$ is treated as a vector. Ai which is a weighted sum becomes vectors of the same dimension too. 


\subsection{Tree Construction for Multiple Sinks}

As an extension to our previous work, we propose to develop an effective data aggregation technique which includes multiple sinks in WSN.

For determining the routing structure towards the sinks, we use the Link Reversal Algorithm. The main objective of this proposed algorithm is to construct and maintain links to multiple sinks to seamlessly aggregate data.

A $R E Q_{\text {sin } k}$ message is flooded periodically by the sink node to the sensor nodes. The request message, $R E Q_{\sin k}$ includes information like Sink ID, Timestamps, Period, Max_Height, Hops and Root ID. Based on the density of deployment of the sensor nodes, the field period is fixed. During this period, the $R E Q_{\text {sin } k}$ message is flooded. The nodes that are at one hop distance from the sink are called as root nodes. The connection or the disconnection status of the sink with its descendent nodes is dependent on the root node. Until the hop of the $R E Q_{\text {sin } k}$ becomes equal to the Max_Height value, the nodes continue to forward the request. Then the nodes start sending its response, $R P Y_{\text {sin } k}$ message to its ascendant node in the tree. Thus, through an established reverse path, a sink oriented tree by height is established.

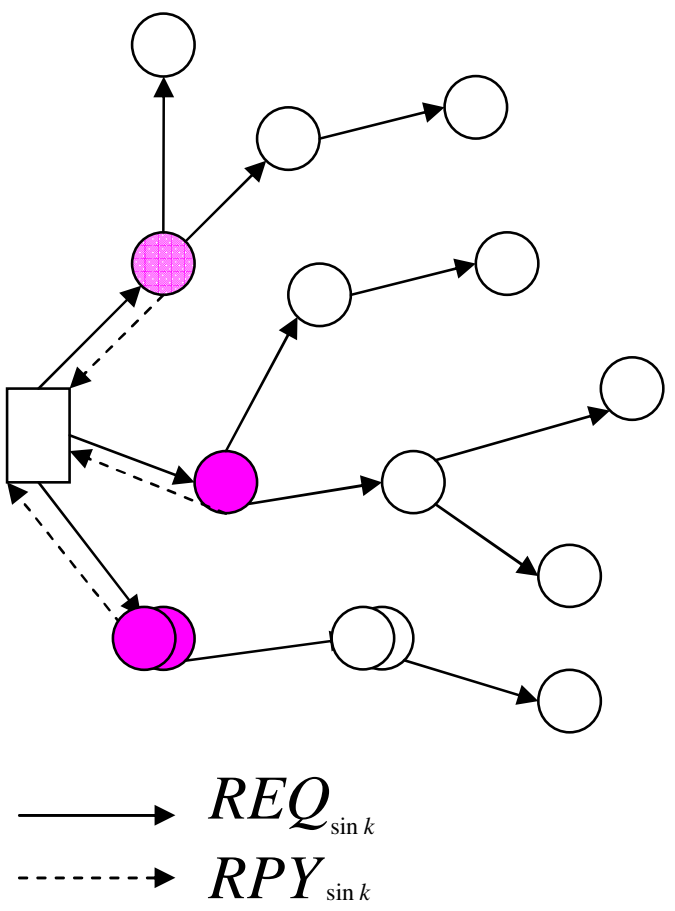

a) Initial sink request and sink reply flooding phase
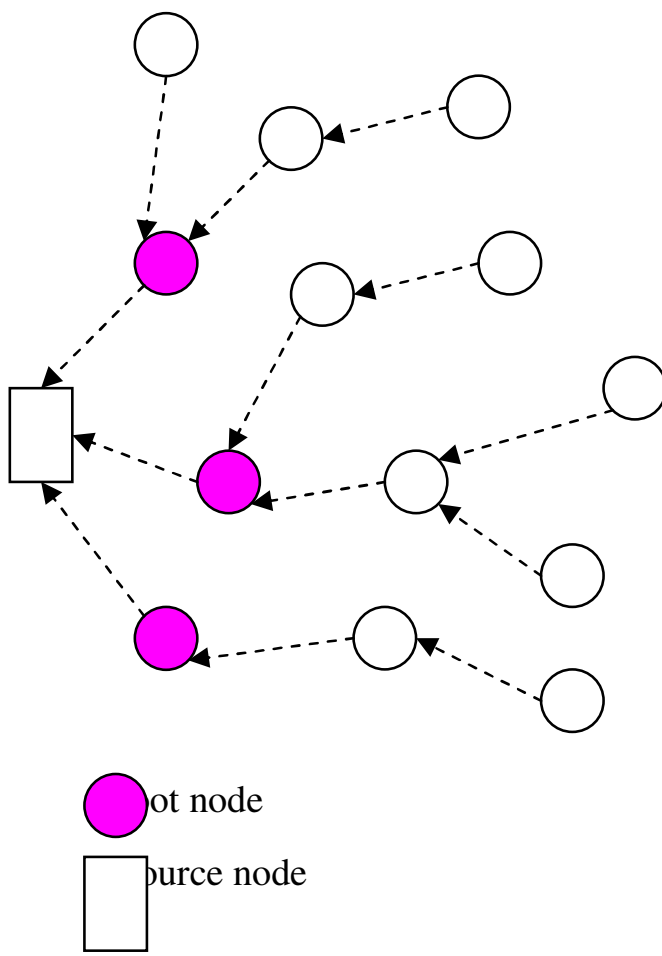

b) Reverse path and sink oriented tree establishment phase 
Another important function of our protocol is the information exchange between sinks. Due to the wide coverage, generally difficulty is faced in developing tree based routing protocols. But due to the merge of independent sink oriented trees our protocol has wider coverage when compared with single sink case. In case a sink sends a request $R E Q_{\sin k}$ to a node which is already a member of some other sink, then the sink id is stored by the node in its memory for passing the data collected to own sink to the new sink which wishes to join it. Hence, to the own sink a query of the sink is sent and in this way the merging of the sink oriented tree of various sinks takes place.

\subsection{Data Collection Scheduling}

For scheduling the data to be aggregated at the sink we develop a scheduling technique for each time interval. We assume that the amount of data to be collected is sufficiently large, and since there are multiple sinks, we split the data into pieces as small as necessary.

In this technique, the entire network is divided into subparts, and then the data is allocated slots for efficient transmission without getting overlapped with the other data. For an interval, [t, $\mathrm{t}+1$, we develop an technique such that basically, for each node $v_{i} \in V$, a duration is scheduled $S_{i}^{t}$ as early as possible without causing any interference.

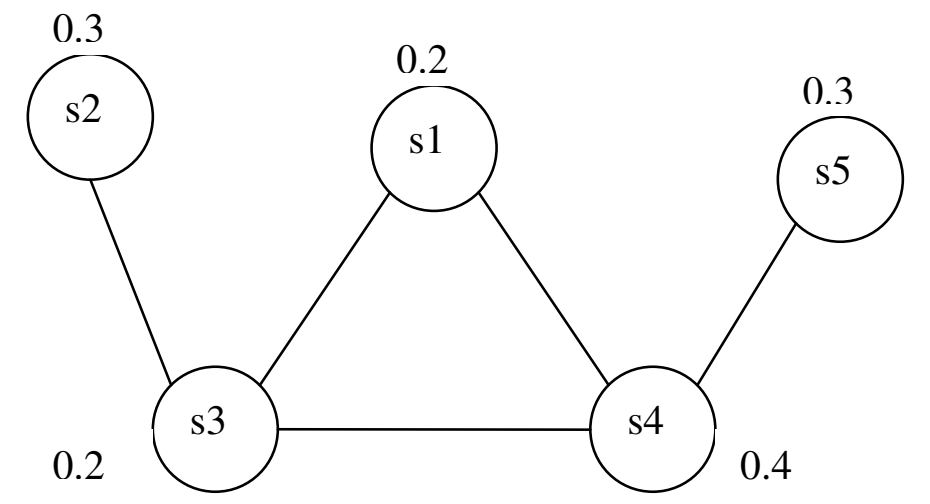

(a) $G_{t}=\left(V_{t}, E_{t}\right)$

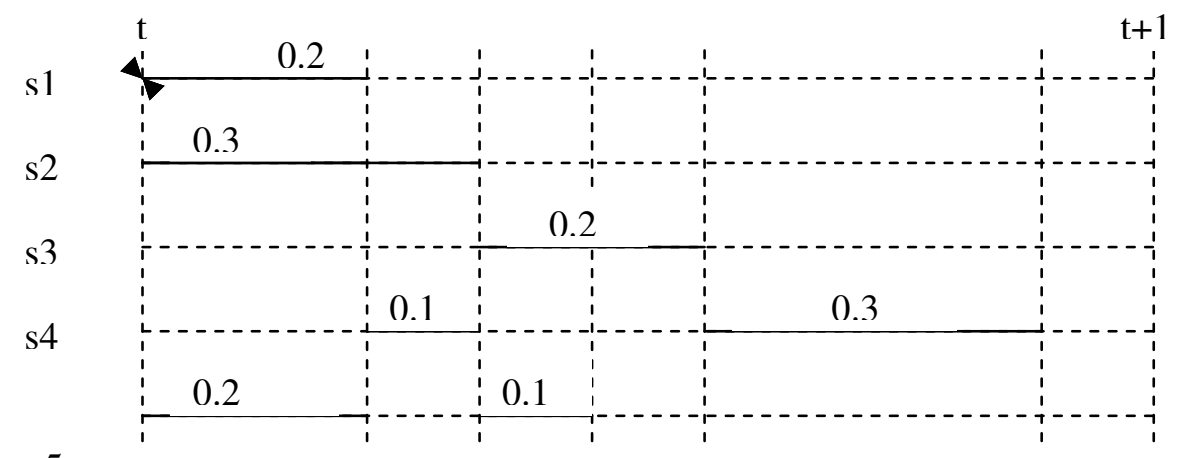

s5

(b) Data Collection Schedule

Fig.2 
Fig 2(a) indicates a link interference graph, $G_{t}$ in which the amount of flow to be transferred over the link is indicated by the number beside each node. For the graph in fig 2(a), a corresponding data collection schedule is shown in fig 2(b). The scheduling starts from time $t$ for the flow at nodes s1 and s2. The scheduling of data transmission for node s 3 starts only after $\mathrm{t}+0.3$ in order to prevent the overlap of its data with that of $\mathrm{s} 2$ leading to interference. For $\mathrm{s} 4$, the scheduling is such that 0.1 is transmitted before the transmission of s 3 starts and 0.3 is transmitted after $\mathrm{t}+0.5$. In a similar process the scheduling of $\mathrm{s} 5$ can be performed.

We have developed a heuristic algorithm for a case in which the network has links with same capacity. In this algorithm, initially based on the distance to the set of sinks, the nodes are divided into layers. The nodes at far distance from the sink transfer their data to the nodes which are comparatively at a smaller distance from the sink, which in turn transfers it to the sink.

In the graph $\mathrm{G}$, let the distance between $\mathrm{p}$ and $\mathrm{q}$ be denoted by $d(p, q)$, which indicates the shortest length in hop counts of the path which connects $p$ and $q$ in the graph G. Let the distance between the node $\mathrm{p}$ and set of nodes $\mathrm{P}$ be denoted by $d(p, P)$ and is defined by $\min _{p \in P} d(p, q)$. Based on the distance of the nodes to the sink set SS, we divide the nodes into different levels. The set of nodes whose distance i to SS is denoted as $\boldsymbol{n}_{i}$. The index of the level that is farthest away from SS is denoted by $n_{\max }$. Using the breadth first search, $n_{i}$ is found where, $\mathrm{i}=1, \ldots ., n_{\max }$. The amount of data at node $\mathrm{p}$ at time $\mathrm{t}$ is denoted by $\operatorname{data}(p, t)$. We consider Func $(t)=\sum_{p \in Q} d(p, S S) \operatorname{data}(p, t)$, a heuristic function which will be equal to zero at the end of the schedule i.e., when all the data have reached the sink. In order to reduce Func(t), the greedy approach maximizes the nodes used for transferring the data at time $t$, by taking into consideration that (i) interference is not created due to transmissions and (ii) transmission to nodes in $\boldsymbol{n}_{i}$ is done by nodes in $\boldsymbol{n}_{i}+1$. This process is repeated until the function $F u n c(t)$ is reduced to zero. The algorithm given below shows the pseudo code of the algorithm. Let $\mathrm{LS}_{\mathrm{t}}$ denote the set of transmitting links at time $\mathrm{t}$.

\section{Greedy BFS based Algorithm}

1: Classify nodes into different levels using breadth first search

2: Let $t=0$

3: while $\operatorname{Func}(t)>0$

do

4: $\quad$ Let $\mathrm{LS}_{\mathrm{t}=} 0$

5: $\quad$ for $i=n_{\max }$ to 1 do

6: $\quad$ sort $\operatorname{data}(p, t)$ in decreasing order, $p \in n_{i}$.

7: $\quad$ for each $p \in \boldsymbol{n}_{i}$ such that $\operatorname{data}(p, t)>0$ do

8: $\quad$ if $p$ has neighbor $q \in \boldsymbol{n}_{i-1}$ such that the link (p,q) does not interfere with any link in $\mathrm{LS}_{\mathrm{t}}$ then 


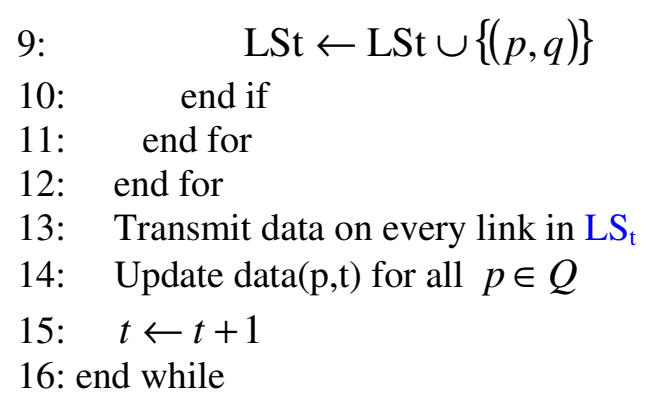

Thus by using this algorithm, the data from numerous nodes can be transmitted to multiple sinks efficiently. Due to the slotting process, the data is split into parts and sent over the channels so as to ensure the complete data transmission without any loss due to overlapping of the data leading to interference in the network.

So the data is transmitted effectively towards the sink through the sink oriented tree established in section 3.2, and as the nodes start aggregating at the higher nodes as it moves nearer to the sink, the data gets compressed as explained in the section 3.1. So at the sink, the data is aggregated such that the compressed data includes slots of data satisfying the sink requirements.

\subsection{Multiple Sink Data Aggregation Algorithm}

1. Sink broadcasts a request message, $R E Q_{\text {sin } k}$ to the sensor nodes periodically based on the interval up to the maximum height.

$$
\text { Sink } \stackrel{R E Q_{\text {sin } k}}{\longrightarrow} \text { sensor nodes }
$$

2. The sensor nodes send the reply message, $R P Y_{\sin k}$ to the sink after the request message reaches the maximum height.

$$
\text { Sink } \stackrel{R P Y_{\sin k}}{\longleftarrow} \text { sensor nodes }
$$

3. The sink oriented tree is established based on the path followed for the transmission of the reply message.

4. The last node in the sink tree starts transmitting the data after computing $\beta_{i j} u_{j}$ using a random number $\beta_{i j}$, to the next possible node in the tree lying towards the sink.

5. The data is transmitted in the slot allocated, so that the overlap between the data being transmitted is prevented.

6. The data gets compressed as it starts getting accumulated from several nodes at the higher nodes.

7. The compressed data is collectively transferred to the sink in interference free slots using the Greedy BFS based algorithm (given in last section). 


\section{Simulation}

\section{1. Simulation Setup}

Multiple Sink Based Compressive Data Aggregation Technique is evaluated through NS2 [18] simulation. A random network deployed in an area of $500 \times 500 \mathrm{~m}$ is considered. We vary the number of nodes as $20,40 \ldots .100$. Initially the nodes are placed randomly in the specified area. The base station is assumed to be situated 100 meters away from the above specified area. The initial energy of all the nodes is assumed as 3.1 joules. In the simulation, the channel capacity of mobile hosts is set to the same value: 2 Mbps. The distributed coordination function (DCF) of IEEE 802.11 is used for wireless LANs as the MAC layer protocol. The simulated traffic is CBR with UDP source and sink. The number of sources is varied from 1 to 5.

Table 1 summarizes the simulation parameters used

Table 1: Simulation Parameters

\begin{tabular}{|l|l|}
\hline No. of Nodes & $20,40, \ldots .100$ \\
\hline Area Size & 500 X 500 \\
\hline Mac & 802.11 \\
\hline Simulation Time & $50 \mathrm{sec}$ \\
\hline Traffic Source & CBR \\
\hline Packet Size & 512 \\
\hline Transmit Power & $0.660 \mathrm{w}$ \\
\hline Receiving Power & $0.395 \mathrm{w}$ \\
\hline Idle Power & $0.335 \mathrm{w}$ \\
\hline Initial Energy & $3.1 \mathrm{~J}$ \\
\hline $\begin{array}{l}\text { Transmission } \\
\text { Range }\end{array}$ & $75 \mathrm{~m}$ \\
\hline No. of Sinks & 2 \\
\hline
\end{tabular}

\section{2. Performance Metrics}

The performance of Multiple Sink based Compressive Data Aggregation (MSCDA) technique is compared with our previous Cost Effective Compressive Data Aggregation CECDA [] protocol, which is based on single sink. The performance is evaluated mainly, according to the following metrics.

Average end-to-end Delay: The end-to-end-delay is averaged over all surviving data packets from the sources to the destinations.

Average Packet Delivery Ratio: It is the ratio of the number of packets received successfully and the total number of packets transmitted.

Energy Consumption: It is the average energy consumed by all the nodes in sending, receiving and forwarding operations

The simulation results are presented in the next section.

\section{3. Simulation Results}

In our experiment, we vary the number of nodes as $20,40,60,80$ and 10 in which the sources are sparsely deployed. 
International Journal of Wireless \& Mobile Networks (IJWMN) Vol. 3, No. 2, April 2011

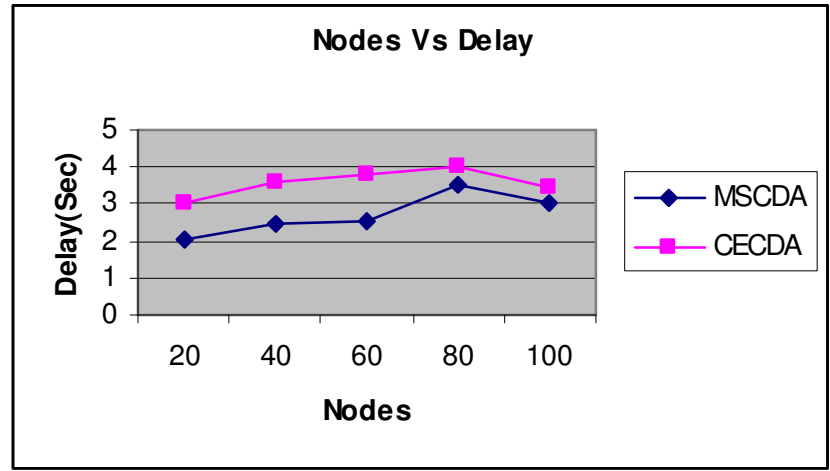

Fig 3: Nodes Vs Delay

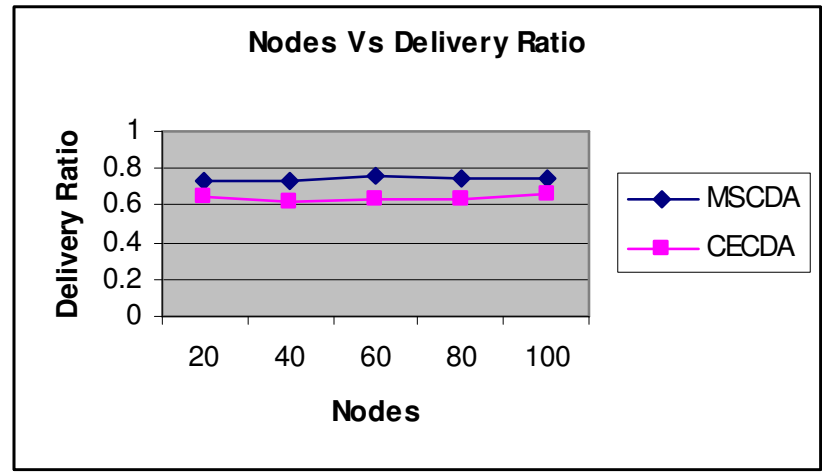

Fig 4: Nodes Vs delivery Ratio

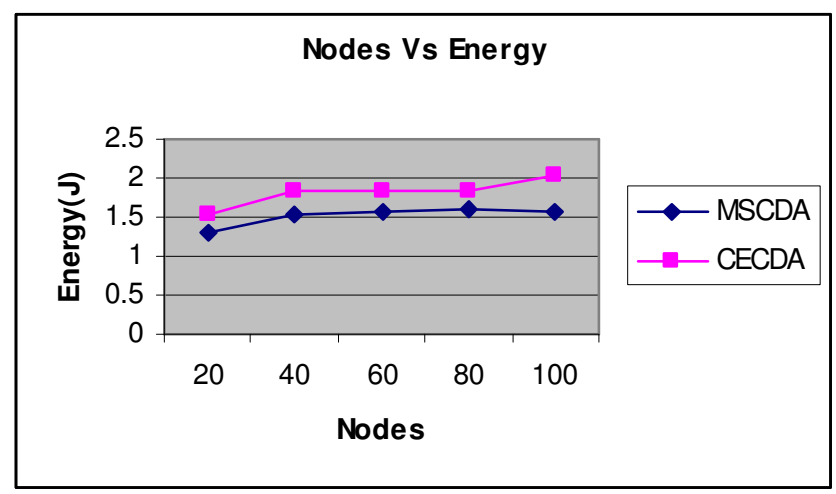

Fig 5: Nodes Vs Energy

Since the aggregation involves compressed data, the delay incurred in sending the data from sensors to the sink, will be significantly reduced.

Fig 3 gives the average end-to-end delay when the number of nodes is increased. From the figure, it can be seen that the average end-to-end delay of the proposed MSCDA technique is less when compared with CECDA. 
Fig 4 presents the packet delivery ratio when the number of nodes is increased. MSCDA achieves good delivery ratio, compared to CECDA. The compressed data aggregation eliminates the packet drops at the intermediate nodes and hence increases the packet delivery ratio.

Fig 5 shows the results of energy consumption when the number of nodes is increased. Compressing the data during data aggregation reduces the number of data packets to be aggregated at the aggregator nodes. Hence the total energy consumption involved in the aggregation process will also be reduced. From the results, we can see that MSCDA technique has less energy consumption when compared with CECDA, since it has the energy efficient tree

\section{Conclusion}

In this paper, we have developed an efficient data aggregation technique for multiple sinks. The data is aggregated based on the assumption that all the sinks are static. We initially develop a sink oriented tree for each sink depending on the requirement of the sink like Timestamps, Interval, Max_Height, Hops, etc. Each sink broadcasts its request to the network and based on the response to the request, the tree is set up. Once the tree is established then the nodes starts transmitting its data to the sinks. The transmission of data is initiated by the nodes which are at a larger distance from the sink, to the following nodes in the tree. The data is transmitted by splitting it into smaller parts. The data is then allocated slots such that the overlapping between the consecutive data is avoided. Then the data is aggregated at nodes nearer to the sink in the compressed form. It is then transmitted similarly to the next level till the sink is reached. This technique is proficient since it allows complete transmission of data even in the presence of multiple sinks and large amount of data. By simulation results, we have shown that our proposed technique achieves good packet delivery ratio with reduced energy consumption and delay.

\section{References}

1] Dorottya Vass and Attila Vidacs, "Distributed Data Aggregation with Geographical Routing in Wireless Sensor Networks", Pervasive Services, IEEE International Conference, 08 August 2007.

2] Cunqing Hua and Tak-Shing Peter Yum, "Optimal Routing and Data Aggregation for Maximizing Lifetime of Wireless Sensor Networks", IEEE/ACM TRANSACTIONS ON NETWORKING, VOL. 16, NO. 4, AUGUST 2008.

3] Tao Cui, Lijun Chen, Tracey Ho, Steven H. Low, and Lachlan L. H. Andrew, "Opportunistic Source Coding for Data Gathering in Wireless Sensor Networks", TAPIA '07 Proceedings of the 2007 conference on Diversity in computing, 2007.

4] Zhenzhen Ye, Alhussein A. Abouzeid and Jing Ai, "Optimal Policies for Distributed Data Aggregation in Wireless Sensor Networks", INFOCOM 2007. 26th IEEE International Conference on Computer Communications. IEEE, 29 May 2007.

5] Bhaskar Krishnamachari, Deborah Estrin and Stephen Wicker, "The Impact of Data Aggregation in Wireless Sensor Networks", ICDCSW '02 Proceedings of the 22nd International Conference on Distributed Computing Systems, IEEE Computer Society Washington, DC, USA @2002.

6] M.Y. Mohamed Yacoab and V. Sundaram, "An Adaptive Traffic Aware Data Aggregation Technique for Wireless Sensor Networks", American Journal of Scientific Research ISSN 1450-223X Issue 10 (2010), pp. 64-77, () EuroJournals Publishing, Inc. 2010. 
International Journal of Wireless \& Mobile Networks (IJWMN) Vol. 3, No. 2, April 2011

7] M.Y. Mohamed Yacoab1, Dr.V.Sundaram2 and Dr.A.Thajudeen, "A Cost Effective Compressive Data Aggregation Technique for Wireless Sensor Networks", International Journal of Ad hoc, Sensor \& Ubiquitous Computing (IJASUC) Vol.1, No.4, December 2010.

8] Feng Wang and Jiangchuan Liu, "Networked Wireless Sensor Data Collection:

Issues, Challenges, and Approaches", IEEE Communications Surveys and Tutorials, 2010.

9] Frank Yeong-Sung Lin, Hong-Hsu Yen and Shu-Ping Lin, "A Novel Energy-Efficient MAC Aware Data Aggregation Routing in Wireless Sensor Networks”, ISSN 1424-8220, Sensors 2009.

10] Emanuele Cipollone, Francesca Cuomo, Sara Della Luna, Ugo Monaco, Francesco Vacirca, "Event detection capabilities of IEEE 802.15.4 Multi-Sink Wireless Sensor Networks", GTTI 2007, Rome (Italy), June 2007.

11] Olga Saukh, Robert Sauter and Pedro Jose Marron, "Convex Groups for Self-organizing Multi-sink Wireless Sensor Networks”, Industrial Electronics, 2009. IECON '09. 35th Annual Conference of IEEE, ISSN: $1553-572 \mathrm{X}, 2009$.

12] Frank Yeong-Sung Lin and Yean-Fu Wen, "Multi-sink Data Aggregation Routing and Scheduling with Dynamic Radii in WSNs", IEEE COMMUNICATIONS LETTERS, VOL. 10, NO. 10, OCTOBER 2006.

13] Khalid N Chaaran, Munzza Younus and Muhammad Younus Javed, "NSN based Multi-Sink Minimum Delay Energy Efficient Routing in Wireless Sensor Networks", European Journal of Scientific Research, ISSN 1450-216X Vol.41 No.3 (2010), pp.399-411, (C) EuroJournals Publishing, Inc. 2010.

14] Luca Mottola and Gian Pietro Picco, "MUSTER: Adaptive Energy-Aware Multi-Sink

Routing in Wireless Sensor Networks", IEEE Transactions on Mobile Computing, 2010.

15] Sixia Chen, Matthew Coolbeth, Hieu Dinh, Yoo-Ah Kim, and Bing Wang, "Data Collection with Multiple Sinks in Wireless Sensor Networks", WASA '09 Proceedings of the 4th International Conference on Wireless Algorithms, Systems, and Applications, Springer-Verlag Berlin, Heidelberg (C)2009.

16] Waleed Alsalih, Selim Akl, and Hossam Hassanein, "Placement of multiple mobile base stations in wireless sensor networks", Signal Processing and Information Technology, 2007 IEEE International Symposium, ISBN: 978-1-4244-1835-0.

17] Zoltan Vincze, Rolland Vida, Attila Vidacs, "Deploying Multiple Sinks in Multi-hop

Wireless Sensor Networks", Pervasive Services, IEEE International Conference, ISBN: 1-4244-1325-7, 2007.

18] Network Simulator, http://www.isi.edu/nsnam/ns 